\title{
Pensiones en México: movilidad social descendente y subjetividad intergeneracional
}

\author{
Pensions in Mexico: descendent social mobility and \\ intergenerational subjectivity
}

\author{
Henio Millán-Valenzuela \\ El Colegio Mexiquense, A.C. \\ hmillan@cmq.edu.mx \\ https://orcid.org/0000-0003-0115-0636
}

Recibido: 04/05/2021 Revisado: 09/06/2021 Aceptado: 22/06/2021 Publicado: 01/09/2021

\section{Resumen}

El sistema de pensiones en México, basado en la capitalización individual, persiste, a pesar de sus evidentes limitaciones para mantener el bienestar que los pensionados registraban en su vida laboral. El objetivo de este artículo es explicar el mecanismo de este descenso social y de las bases subjetivas que lo legitiman. Se emplea un modelo matemático simple para estimar la pensión de un contribuyente promedio. Los principales resultados son: a) el retiro en México trae consigo un sustancial descenso económico de los pensionados; b) este descenso frecuentemente acarrea caídas en la pobreza para quienes no eran pobres durante su vida laboral. Y se concluye que una explicación razonable para este sistema de pensiones se puede encontrar tanto en los cambios de criterios de justicia distributiva como en las subjetividades intergeneracionales.

\section{Palabras clave}

México, pensiones, movilidad descendente, subjetividad intergeneracional, criterios, justicia distributiva.

Forma sugerida de citar: Millán-Valenzuela, H. (2021). Pensiones en México: movilidad social descendente y subjetividad intergeneracional. Universitas-XXI, 35, pp. 207-227. https://doi.org/10.17163/uni.n35.2021.10 


\begin{abstract}
Mexican system of pension, based on "pay-as-you go" (individual capitalization) persists, though its obvious limitations for keeping the well-being pensioners had during their labor life. The objective of this article is to explain the mechanism behind this social descendent and its subjective legitimacy bases. A simple mathematical model is used for estimating the hypothetical pension fund that an average contributor in Mexico would be able to get. The main results are: a) in Mexico retirement brings a substantial economic descent of pensioners; $b$ ) frequently such descent drives to poverty falls for those who were not poor during their labor life. And it concludes that a reasonable explanation for the persistence of this pension system can be found in, both, the changes of distributive justice criteria and in the intergenerational subjectivities
\end{abstract}

\title{
Keywords
}

Mexico, pensions, descendent mobility, intergenerational subjectivity, criteria, distributive justice

\section{Introducción}

En septiembre de 2020, el gobierno mexicano presentó una iniciativa de enmienda ante el Congreso de la Unión para modificar su sistema de pensiones. La iniciativa es de reforma paramétrica y no estructural, en el sentido de que pretende alterar los valores de los principales coeficientes que determinan el acceso y el monto de los fondos de pensiones, pero deja intacto el esquema de capitalización individual. Ha sido bien recibida. Sin embargo, sorprende que mantenga el encuadre operativo asociado al espíritu y la lógica neoliberal, dado que la nueva administración se considera de izquierda y es abiertamente opuesta a este pensamiento. Incluso va en contra de las corrientes contemporáneas, que se inclinan por dejar atrás este esquema de pensiones o subordinarlo a otros que enfatizan la solidaridad.

Es decir: las transferencias no condicionadas y los programas sociales no intermediarios "se vendieron" como uno de los principales puntos de ruptura con el pasado inmediato. Este "nuevo" enfoque se presentó como una política de derechos, de acuerdo con el enfoque en boga (Filgueira et al., 2006). El mensaje fue inequívoco: la guía de la política social sería la desmercantilización de las dimensiones más importantes de la vida; y, sobre todo, apartándolos de la lógica meritocrática. Sin embargo, la reforma de las pensiones parece 
mantener el espíritu neoliberal. Esto facilita que la economía siga dominando el espacio de las políticas sociales, como lo hizo en las últimas décadas, aunque todo parecía indicar una creciente autonomía frente a las relaciones de mercado y una protección frente al predominio de la política económica.

Este hecho es lo que ha motivado este artículo. La persistencia de la influencia de la visión neoliberal en uno de los ámbitos históricamente más sensibles - el bienestar de las personas mayores los anciano- sugiere mutaciones que exceden con mucho el alcance del trabajo gubernamental. Señala un cambio en la subjetividad de los actores sociales sobre lo que es justo. Si bien antes se esperaba una respuesta de intervención para evitar cualquier amenaza peligrosa, hoy en día dejar a la deriva las condiciones de vida de las personas mayores es visto casi como "normal" por la nueva generación. Este cambio parece ser un rasgo común en el mundo occidental, pero la indiferencia se vuelve dramática en países subdesarrollados como México, porque conlleva la posibilidad de sufrir un profundo declive económico y social y, con frecuencia, el empobrecimiento de las condiciones de existencia. Otros han estudiado la relación entre pensionados y pobreza (Rodríguez, 2016), pero no la movilidad social descendiente (a veces la caída en la pobreza) que puede producir la jubilación. El objetivo es examinar esta posibilidad e indagar sobre subjetividades que permitan este cambio.

El artículo está dividido en cuatro secciones. La primera presenta la reciente propuesta del gobierno mexicano que pretende modificar el sistema de pensiones, de manera contraria a las principales tendencias internacionales. La segunda describe el modelo matemático detrás de los cálculos que utilizan las autoridades mexicanas para estimar las pensiones individuales. La tercera sección presenta y discute los resultados de aplicar ese modelo a un contribuyente promedio, para explorar la movilidad social de los pensionados luego de su retiro de la vida laboral. La cuarta examina un elemento fundamental para pensar en la vejez y las pensiones: los criterios de justicia distributiva en las sociedades premodernas, modernas y posmodernas.

\section{La propuesta del nuevo gobierno y las tendencias internacionales actuales}

El sistema de capitalización individual fue la respuesta de la política neoliberal al problema del financiamiento de las pensiones. La forma habi- 
tual de describir este problema consiste en invocar varios indicadores que apuntan al mismo mensaje: el envejecimiento de la población. La extensión de la esperanza de vida y la reducción de las tasas de mortalidad resultó en un menor número de contribuyentes, reales y potenciales, lo que a su vez afectó la capacidad del Estado para financiar los crecientes montos de las pensiones. Las siguientes cifras describen esta situación para México:

\section{Figura 1}

\section{Población en edad laboral para adultos mayores}

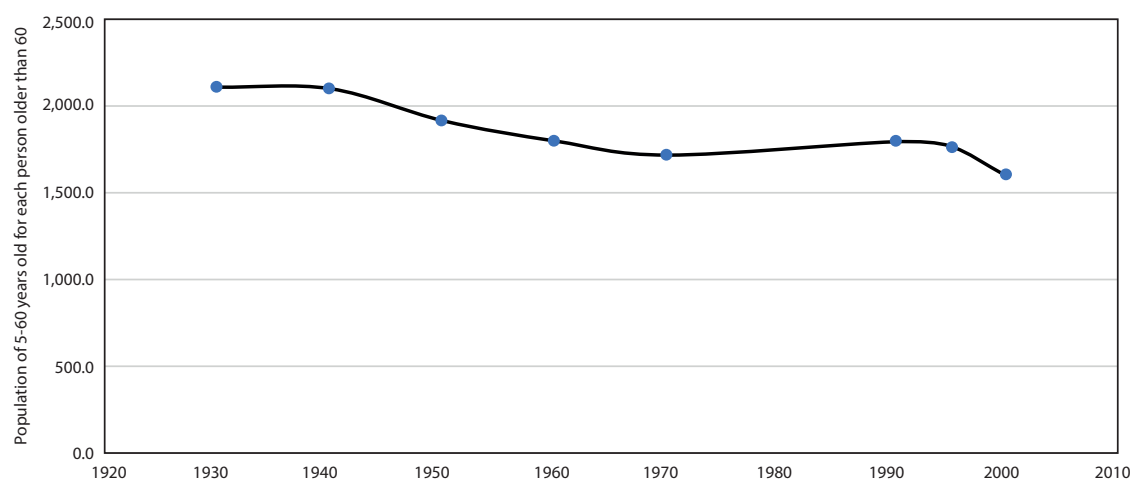

Fuente: INEGI: Estadísticas socioeconómicas 1930-2000.

\section{Figura 2}

\section{Contribuyentes por cada pensionado (Gobierno Federal)}

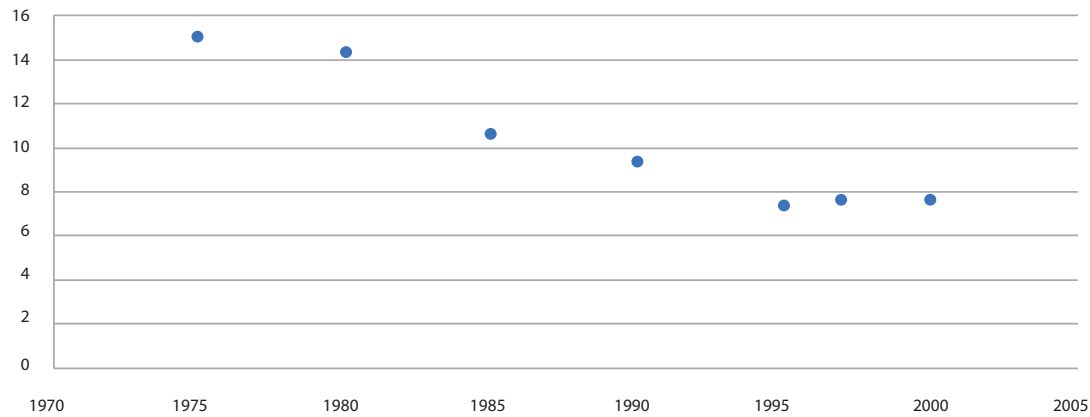

Fuente: INEGI: Estadísticas socioeconómicas. 
Como consecuencia del envejecimiento, las finanzas públicas se veían amenazadas por una presión más fuerte: si a través de los trabajadores activos no se pueden cubrir los gastos de los pensionados, el Estado debería ser responsable de cubrir la diferencia. Pero, en lugar de emprender una reforma fiscal, el Estado respondió en julio de 1997 con un sistema de capitalización individual (Ramírez, 2017). La responsabilidad se transfirió al trabajador, que ahora debería ser responsable de sus propios ahorros para la jubilación.

Esta fue la forma de actualizarse a las tendencias neoliberales: en 1981, Chile cambió su sistema de pensiones de un sistema de reparto a uno nuevo de capitalización individual. Otras naciones latinoamericanas, entre ellas México, siguieron el ejemplo: Bolivia (1997); El Salvador (1998) y República Dominicana (2003). Compartían una característica común: la reforma instaló la capitalización individual como único sistema de pensiones. En otros, como Perú (1993) y Colombia (1994), la gente debe elegir entre este y el antiguo sistema. Lo mismo sucedió en Argentina (1994), pero con una diferencia importante: la incorporación de un mecanismo de solidaridad como base para todo el sistema. En Costa Rica, Panamá y Uruguay, ambos fueron complementarios (Arenas, 2019).

México es el único país con un esquema de capitalización único. Aunque supuso un alivio para las finanzas públicas (OCDE, 2016), está lejos de haber resuelto por completo los problemas originales. Y ha generado otros: Villarreal y Macías (2020) señalan inestabilidad financiera; Martínez-Preece et al. (2019) la alta volatilidad de las rentabilidades; y Durán y Peña (2011) el impacto de las comisiones en la tasa de reemplazo. ${ }^{1}$ Pero son dos los más importantes: la baja cobertura (Alonso et al., 2014) y el magro monto de la pensión - para quienes logran acceder a alguna. Estas son las mayores preocupaciones para casi todos los países de América Latina (BID, Banco Mundial \& OCDE, 2015) y las cifras son muy similares. Por ejemplo, en México la cobertura es del $56 \%$; pero solo el $22 \%$ tendrá un saldo final suficiente para pagar la renta vitalicia. El resto (34 \%) deberá recurrir a una Pensión Garantizada (SHCP, 2020), apenas superior a la línea de pobreza y respaldada tanto por el monto acumulado por el pensionado como por las aportaciones gubernamentales. ${ }^{2}$

1 Para los efectos de la reforma de 1997 ver Herrera y Velázquez (2018).

2 La Pensión Garantizada se otorga a quienes cumplen con los requisitos de edad (sesenta y cinco años) y 1250 semanas de cotización, pero el saldo final de su cuenta de capitalización individual es insuficiente para contratar un seguro de renta vitalicia. 
Consecuencias similares en los países de América Latina provocaron que muchos sistemas de pensiones se alejen del esquema de capitalización solitaria y vuelvan al sistema solidario, que, junto a las pensiones no contributivas, da un piso mínimo. Las cuentas individuales correspondientes ahora son complementarias y opcionales, pero ya no la principal forma de funcionamiento (Arenas, 2019). Lo mismo sucedió en Europa del Este (Ortiz et al., 2018).

En septiembre de 2020, el gobierno mexicano presentó una iniciativa ante el Congreso con la intención de modificar el sistema de pensiones mexicano, a pesar de las tendencias actuales en América Latina, hacia la restauración tanto del espíritu como de los mecanismos de solidaridad, la propuesta mantiene el sistema de capitalización como principal protagonista. El único componente solidario consiste en la parte no contributiva, que está lejos de representar una parte significativa del esquema global. Las modificaciones son paramétricas: se alteran los coeficientes de capitalización, pero sin cambiar el mecanismo de financiación. Las mutaciones más importantes son la reducción de las semanas de cotización necesarias para acceder a una pensión (de 1250 a 1000, después de una reducción temporal a 750); encabezando la comisión de gestión privada; el aumento de la Pensión de Garantía y el aumento del 6,5 al $15 \%$ de la tasa de cotización. El peso del aumento recae exclusivamente en los empleadores, quienes, en términos generales, se harán cargo de los ingresos más altos: aproximadamente el $30 \%$ de la población. El gobierno, por su parte, combina en un solo fondo su antiguo aporte a la cuenta de jubilaciones y cesantías en vejez y la cuota social; pero ahora este fondo adicional se otorga solo a los empleados que ganan hasta cuatro Unidades de Medida y Actualización (UMAS). ${ }^{3}$ Antes de estos cambios, la contribución del gobierno beneficiaba a toda la fuerza laboral formal y se otorgaba cuota social a los salarios por debajo de los dieciséis UMAS. Sin agregar un solo peso al fondo de pensiones, distribuye el monto entre los trabajadores más pobres, ya que el sector privado se relaciona con los empleados de mayores ingresos. ${ }^{4}$

3 La Unidad de Medida y Actualización es la medida que se utiliza para indexar algunas variables según la inflación. Reemplazó el salario mínimo una vez que se decidió emprender una estrategia de recuperación.

4 Para una descripción detallada de la propuesta de gobierno ver Clavellina (2020). 
Varios problemas surgen de esta reforma. El más importante es la renuencia a volver a algún esquema solidario. La relevancia que conserva la capitalización individual denuncia el apego tanto al espíritu como al modelo neoliberal. El gobierno encuentra difícil separarse de ello, a pesar de su insistencia en acusar a esta política de ser la bête noir de México. El segundo problema es que la iniciativa toma los aspectos centrales de las propuestas de los empresarios, que se han articulado para no tocar significativamente los salarios, en un contexto de estrategia gubernamental para recuperar el salario real. El tercer problema es que no incluye el contingente de trabajadores informales, que representa a la mayoría de los empleados (INEGI, 2020). Además, comienza con un supuesto poco realista: la dinámica de las semanas de cotización, a partir de las 750 semanas y luego los incrementos anuales de veinticinco, supone que la economía se volverá cada vez más formal. Deja atrás el carácter estructural de esta distribución de actividades económicas y mano de obra. En este sentido, opera bajo la misma hipótesis del modelo de capitalización individual.

\section{Materiales y métodos}

La ruptura unilateral de los lazos vinculantes es uno de los ingredientes más perversos para explicar la pobreza de los pensionistas en los países subdesarrollados. Aunque es una característica compartida con las sociedades ricas, cuando se combina con bajos ingresos laborales, el sistema de capitalización individual no produce una vida pacífica de jubilación. En estos últimos países, el contexto es diferente: la gente gana altos ingresos y, sobre todo, trabaja en economías fuertes y formales. Esto no sucede en sociedades de ingresos medios, con altos niveles de pobreza y economías predominantemente informales:

Está documentado que los esquemas de capitalización individual ofrecerían buenos resultados para los hombres, como trabajadores formales de las áreas urbanas, con ingresos elevados y trabajos estables, que contribuyen al sistema durante la mayor parte de la vida laboral (con altas densidades de cotización). Sin embargo, estos trabajadores no son representativos de los países de la región. Por una parte, la creciente e importante informalidad del mercado laboral impide la extensión de la cobertura de los sistemas de pensiones. Además, entre los trabajadores pertenecientes al mercado laboral formal 
existe una gran proporción de trabajos inestables con bajas densidades de cotización... (Arenas, 2019, p.132)

En México, como en el resto de Latinoamérica, no se cumplen los supuestos del esquema de capitalización. Los ingresos no son elevados, la mayoría de la población activa no tiene puestos de trabajo estables y estos no se despliegan en el ámbito de la formalidad. La primera consecuencia es que la cobertura es muy baja: solo una parte muy exigua del contingente de trabajadores accederá a una pensión debido a la baja probabilidad de que cumplan con los requisitos para el período de cotización. Los trabajadores entran y salen de los mercados formales e informales, lo que se traduce en una baja densidad de cotización. En segundo lugar, el monto que se podrá acumular durante la vida laboral formal será insuficiente, debido al bajo salario derivado del fracaso neoliberal para lograr niveles satisfactorios de competitividad a través de la productividad y el progreso técnico. El salario se convirtió en la variable de ajuste. Y, en tercer lugar, porque las pensiones serán tan escasas que, para un trabajador medio, puede significar caer en la pobreza, incluso cuando no ha sido pobre durante su vida laboral. En otras palabras, descenderá en la escala socioeconómica.

Para demostrar esta proposición, desarrollamos un ejercicio que utiliza un contribuyente promedio de México. El cálculo de la pensión se basa en el algoritmo matemático de la calculadora de la Comisión Nacional del Sistema de Ahorro para el Retiro (CONSAR, 2018). Fue alimentado por los datos reportados por CONSAR y SHCP (Secretaría de Hacienda y Crédito Público).

La calculadora de CONSAR está diseñada para estimar el monto mensual de una pensión, en dos pasos. El primero calcula el saldo final de la cuenta de ahorro para el retiro; el segundo estima el monto mensual de la pensión.

\section{Primer paso: balance final}

$$
S_{f}=S_{i}\left(1+r^{(m)}\right)^{n}\left(1-c^{(m)}\right)^{n}\left[d\left(A_{0}+A_{v}+C_{s}\right)\left[\frac{\left(1+r^{(m)}\right)^{n}\left(1-c^{(m)}\right)^{n}-1}{\left(1+r^{(m)}\right)\left(1-c^{(m)}\right)-1}\right]\right]
$$


Donde:

$\mathrm{S}_{\mathrm{f}}$ : balance final; $\mathrm{S}_{\mathrm{i}}$ : saldo inicial, que se supone que es el salario medio de contribución; $\mathrm{r}^{(\mathrm{m})}$ : tasa de rendimiento mensual, en términos reales, $r^{(m)}=\left(1+r_{\text {annual }}\right)^{\frac{1}{12}}-1$ y r: tasa de rendimiento anual real. El modelo asume el valor del 5,81\%, que es el promedio histórico desde que comenzó el esquema en 1997.

Por otro lado, $\mathrm{c}^{(\mathrm{m})}$ representa una comisión mensual y se calcula de la siguiente manera: $c^{(m)}=\frac{\text { annual commision over balance charged by Afore }}{12}{ }^{5}$ La comisión anual en consideración es la que arroja el sistema: 0,92\%.

Por otro lado, $d$ es la densidad de cotización: el tiempo que el trabajador ha aportado como proporción de su vida laboral. Se asigna un valor del $50,1 \%$, que es lo que reporta la SHCP (2020) como promedio, y del $80 \%$ para quienes alcanzan las 1250 semanas de cotización y, por tanto, pueden acceder a una pensión. $A_{o}$ es la cotización obligatoria: según la legislación equivale al 6,5\% del salario base de cotización. El promedio fue de 11886 pesos mexicanos (MX\$) a febrero de 2020, reportado por el Instituto Mexicano del Seguro Social (IMSS, 2020).

$A_{v}$ es el aporte voluntario y corresponde al ahorro voluntario individual. Se estimó como la relación aritmética entre el ahorro voluntario total (MX \$ 92 billones) y el número de cuentas: 65,9 millones (CONSAR, 2020). $C_{s}$ es la cuota social. Es otorgado por el gobierno (además del aporte) a cada individuo con un salario menor a dieciséis UMA (Unidad de Medida y Actualización). Disminuye a medida que aumenta el salario. El valor correspondiente al salario base promedio de cotización es de 175.9 MX\$ mensuales. Y $n$ es el número de meses de cotización hasta la jubilación.

\section{Segundo paso: la pensión mensual}

El monto de la pensión mensual se calcula de la siguiente manera:

$$
\text { Monthly pension }=\frac{S_{f}}{12 * U R V}
$$

5 La Administradora de Fondos de Ahorro para el Retiro (AFORE) cobra una comisión sobre el monto acumulado del ahorro del trabajador por el manejo de la cuenta. 
Donde: $U R V$ es la Unidad de Anualidad: el monto necesario para financiar el pago de cada peso de la pensión anual al trabajador jubilado. La CONSAR publica el valor de estos factores semanalmente. Se eligió la segunda semana de febrero de 2020.

\section{Análisis y resultados}

\section{Estimaciones fácticas e hipotéticas}

Los resultados del ejercicio anterior se describen en la tabla 1:

Tabla 1

\section{Pensión real y simulada para un contribuyente medio}

\begin{tabular}{|c|c|c|c|}
\hline Items & \multicolumn{2}{|c|}{ Real } & \multirow{2}{*}{$\begin{array}{c}\text { Hipotética } \\
\text { (B) }\end{array}$} \\
\hline Escenario & (A) & (C) & \\
\hline Semanas de contribución & 797 & 1250 & 797 \\
\hline $\mathrm{S}_{\mathrm{i}}$ Salario base de contribución (\%) & \multicolumn{3}{|c|}{11886} \\
\hline r: Tasa anual de rendimiento $(\%)$ & \multicolumn{3}{|c|}{5.81} \\
\hline c: comisión anual $(\%)$ & \multicolumn{3}{|c|}{0.92} \\
\hline d: Densidad de contribución (\$) & 50.1 & 80.0 & 50.1 \\
\hline Ao: Contribución mandataria (\$) & \multicolumn{3}{|c|}{772.59} \\
\hline Av: Contribución voluntaria (\$) & \multicolumn{3}{|c|}{1396} \\
\hline $\mathrm{S}_{\mathrm{c}}:$ Cuota social $(\$)$ & \multicolumn{3}{|c|}{175.91} \\
\hline$S_{f}:$ Balance final de la cuenta $(\$)$ & 341089,08 & 1042207.40 & 341089.08 \\
\hline URV (puntos) & \multicolumn{3}{|c|}{17.75} \\
\hline $12 * \mathrm{URV}$ & \multicolumn{3}{|c|}{213.5} \\
\hline Pensión Mensual & Negada & 4891.86 & 1600.98 \\
\hline Expectativa de vida a 65 años: años & \multicolumn{3}{|c|}{18.2} \\
\hline Expectativa de vida a 65 años: meses & \multicolumn{3}{|c|}{$218-4$} \\
\hline Ingreso mensual & 1561.70 & 4891.86 & 1600.99 \\
\hline \multicolumn{4}{|c|}{ Línea de pobreza (febrero 2020) } \\
\hline Rural \$ & \multicolumn{3}{|l|}{2082} \\
\hline Urbana \$ & \multicolumn{3}{|l|}{3207} \\
\hline
\end{tabular}

Fuente: Elaboración del autor con datos de CONSAR (2020); IMSS (2020) y SHCP (2020). 
El ejercicio se realizó bajo tres escenarios para el mismo contribuyente. El escenario (A) refleja las condiciones fácticas del promedio de semanas de contribución (797); el segundo (B) realiza una simulación con el mismo número de semanas, pero incluye una decisión hipotética: el gobierno opta por dar una pensión de acuerdo con el saldo final acumulado de la cuenta, aun cuando no se alcance el requisito de cotización de 1250 semanas. Y la tercera (C) considera una situación en la que, según la legislación vigente, se concede la pensión porque se cumple ese requisito. El cambio paramétrico más importante, en este sentido, es el aumento de la densidad de contribución. En todos los casos, el salario base de cotización promedio que reporta el Instituto Mexicano del Seguro Social (IMSS) para febrero de 2020 es de 11886 pesos.

\section{Pobreza y movilidad descendiente en el sistema mexicano de pensiones}

Veamos el primer caso (escenario A). Es obvio que este trabajador representativo no es un hombre pobre, en la medida en que sus ingresos superan con creces las líneas de pobreza, tanto para las líneas rurales como urbanas, que sirven de umbral para separar por ingresos a las personas que son y no pobres. ${ }^{6}$ Sin embargo, en virtud del carácter predominantemente informal del mercado, el período medio de cotización de un trabajador es de 797 semanas, equivalente a una densidad del 50,1\%.7 Si esta estadística se aplica a nuestro trabajador promedio; $y$, además, consideramos como condicional el monto acumulado a la edad de sesenta y cinco años y las variables reportadas por CONSAR - rendimiento histórico promedio, comisión corriente cobrada por las AFORES (Administradoras de Fondos de Retiro), participación social provista por el gobierno mexicano, y el ahorro voluntario promedio - el resultado arroja un saldo final de la cuenta de 341089 MX\$. Es obvio que no se alcanza el número de semanas de cotización necesarias para tener derecho a acceder a una pensión (1250 semanas). Este contribuyente no tendrá derecho a percibir una pensión; es decir, tendrá como respuesta una "negativa" cuando solicite formalmente una pensión por no alcanzar el período de cotización exigido.

6 La línea de pobreza es el $27 \%$ del salario del contribuyente medio.

7 La vida laboral media es de treinta años, equivalente a 1560 semanas. 
En este caso, la AFORE realizará un único pago por el saldo final de la cuenta. A los sesenta y cinco años, una persona mexicana tiene una esperanza de vida de poco más de dieciocho años, equivalente a 218,4 meses. Al dividir el monto final por esta cantidad, el monto mensual que este contribuyente promedio puede dedicar a su manutención será de MX \$1,561; esto es incluso menor a lo que proporcionaría la pensión hipotética.

Pero supongamos que la autoridad ignora este requisito y proporciona una pensión hipotética (escenario B), con base en el monto final acumulado. ${ }^{8}$ Como el lector puede ver, este escenario hipotético no se basa en la propuesta del gobierno mexicano de acceder a una pensión garantizada con 750 semanas iniciales para el primer año. La primera razón es que después de este período, el requisito de semanas aumenta en veinticinco por año. Es necesario un año y medio para mantener fuera, nuevamente, al contribuyente promedio. El segundo es la política de austeridad mostrada por el gobierno, reacio a gastar en otra cosa diferente a la energía y los programas sociales clientelares. Esto explica la acogida dada a la propuesta de los empresarios que, para todo efecto práctico, conlleva la ampliación del mecanismo neoliberal de financiación de las pensiones. Por lo tanto, podemos especular sobre lo que sucedería si el gobierno decidiera "vender" una anualidad por el precio de la cantidad acumulada. La pensión sería de MX\$1601: 23 \% por debajo de la línea de pobreza rural; y $50 \%$ debajo de la línea urbana.

El mensaje es inequívoco: en ambos escenarios (pensión hipotética y negativa) el trabajador promedio caerá en una pobreza extrema, sin haberlo sido pobre durante su vida laboral. Y esto, en realidad, no describe la realidad de los trabajadores mexicanos. El trabajador en cuestión refleja un salario medio, lo que esconde la enorme dispersión salarial. La mayoría de los trabajadores ganan mucho menos que esa cantidad. La Encuesta Nacional de Ocupación y Empleo (INEGI, 2020) revela que el 72 \% de la población ocupada recibe un ingreso por debajo de ese salario base promedio de cotización. Sabemos que cuanto menor es el ingreso, mayor es la probabilidad de trabajar en la economía informal. Por tanto, también es más probable que la densidad de cotización sea menor para ingresos inferiores que la del tra-

8 Como puede ver el lector, el escenario hipotético no se basa en la propuesta del gobierno mexicano de acceder a una pensión garantizada con 750 semanas iniciales para el primer año. Después de esto, el requisito de semanas aumenta en veinticinco por año. En este sentido, después de un año y medio, el trabajador medio no podrá acceder a este beneficio. Entonces, podemos especular sobre lo que sucedería si el gobierno decide "vender" una anualidad por el acumulado 
bajador representativo. Esto aumenta la probabilidad de que los trabajadores no pobres, pero de bajos ingresos, caigan en la pobreza. Y aquellos que son pobres, experimentarán una situación más severa durante el período de jubilación. Un estudio reciente (Sánchez \& Rodríguez, 2020) concluye que la expansión de las pensiones no contributivas ha tenido muy poco impacto en el alivio de la pobreza.

La tercera fase del experimento (C) simula el caso en el que el contribuyente promedio llega al período de cotización. Este supuesto conlleva cambios que se manifiestan en la densidad de cotización, el saldo final y el monto de la pensión, como se refleja en la tabla 1. Hay un efecto importante: a diferencia del caso promedio real, nuestro contribuyente no caerá en la pobreza. De hecho, la pensión mensual que recibiría es un $53 \%$ más alta que la línea de pobreza urbana; y más del doble (135\%) de la rural. Sin embargo, es inevitable que sufra una movilidad social a la baja: la pensión representará el $41 \%$ del salario que, en promedio, percibió durante su vida laboral. Los sistemas tradicionales de prestación, como el amparado por la ley de 1973, contemplaban una base mínima, equivalente al $75 \%$ del salario promedio, y cinco puntos porcentuales de aumentos, a partir de los sesenta años, hasta llegar al $100 \%$.

Otra forma de verlo: aunque en el momento de la jubilación se hacen los mayores gastos (hijos, hipoteca, coche, etc.), esta movilidad social descendente sugiere privaciones no sufridas durante la vida laboral.

\section{Discusiones y conclusiones}

En esta sección se ensaya una explicación de la nueva "normalización de la subjetividad" que parece romper con el pasado y correlacionar con los tiempos actuales. Imaginemos dos situaciones. El primero describe un grupo escolar que asiste a un curso impartido por algún profesor. Imagínense dos estudiantes cuyas actitudes son diametralmente diferentes: el primero es responsable, cumple con todos sus trabajos, asiste a clases invariablemente, está al día con sus lecturas a tiempo y estudia permanentemente para los exámenes. El segundo, sin embargo, es algo vago, no cumple con sus tareas y lecturas, pone cualquier excusa para no asistir a clase, y cuando lo hace, está medio dormido y con las últimas señales de la borrachera de la noche anterior. La pregunta es la siguiente: ¿deberían ambos estudiantes tener la 
misma nota? Si justicia significa "igualdad", la respuesta sería sí. Pero no era este tipo de igualdad en la que pensaban los promotores de la modernidad, sino una igualdad en la que, a partir de circunstancias similares, el esfuerzo se convirtió en la única justificación de la diferenciación social. En este caso, la respuesta a la pregunta sería no. El primer estudiante fue dedicado y realizó un mayor esfuerzo, por lo que debería obtener una nota más alta que el segundo, que se desempeñó mal y sin esfuerzo. Esta respuesta corresponde al criterio meritocrático de justicia distributiva.

La segunda situación alude a una familia típica: además de los padres, las madres y algunos hermanos y hermanas, que contribuyen a la economía familiar, encontramos niñas y niños pequeños, así como abuelos y abuelas ancianos. Este último grupo, por su edad, no aporta a ese núcleo social. La pregunta, entonces, es la siguiente: ¿es correcto negar comida, albergue y vestimenta a los infantes y ancianos, por no contribuir como el resto de la familia? La respuesta obvia es no. El estricto criterio meritocrático propondría, no solo en el aula, sino también en el lugar de trabajo y otros espacios, que todo el mundo debería recibir algo, según su aportación. Ese fue el espíritu que impregnó la idea de socialismo de Marx. ${ }^{9}$ En ese sentido, ni los niños/las niñas ni los adultos/las adultas deben recibir parte alguna de lo que resulta del esfuerzo de los demás.

Esta idea nos asusta, porque detrás de ella se esconde un criterio distributivo diferente que responde a un tipo de sociedad también diferente. El criterio distributivo es solidario porque la familia es el ejemplo más elocuente del tipo social de naturaleza comunitaria. Las comunidades son extrapolaciones de la familia, porque las interacciones sociales se rigen por vínculos personales y los intercambios se rigen por razones no interesadas. Lo contrario sucede en las sociedades modernas, en las que las interacciones surgen y resultan como un subproducto de la búsqueda de intereses personales. Hacemos amigos en el trabajo porque llegamos al lugar de trabajo para recibir un salario, mientras que la organización establece una relación con nosotros porque tienen interés en el potencial laboral que representa-

9 Sin embargo, es necesario señalar que Marx aclara que este es el ideal distributivo en el socialismo: lo que todos reciben debe ser acorde con su contribución. (Diferente del ideal comunista: lo que todos reciben está de acuerdo con sus necesidades). Pero en esta etapa, será necesario canalizar recursos para la administración estatal, la inversión pública, que hará que las personas reciban, individualmente, una cantidad menor a lo que aportaron. Esta fue la crítica a La Salle en la Crítica de Marx al Programa de Gotha 
mos para sus actividades. Construimos relaciones con nuestros compañeros de clase, porque tenemos el interés común de obtener un título universitario, y así sucesivamente. Sin embargo, en las familias, como en las comunidades, se establece lo que Durkheim (1964) llamó "solidaridad mecánica": ayudarse unos a otros se da por sentado simplemente porque el vínculo personal lo exige. La "solidaridad orgánica" es propia de la sociedad moderna y opera en la medida en que cumplimos una función necesaria para el organismo; es decir, en la medida en que seamos útiles para su funcionamiento. Las personas en condición de niñez todavía no son útiles; las que están en la vejez ya no lo son. Si aplicamos los criterios meritocráticos del sistema capitalista, simplemente descartaríamos a esos grupos. En cambio, es el criterio distributivo solidario el que los protege de esa lógica mercantil. No es casualidad que Marx postuló este criterio durante la etapa comunista, después de la expiración de la etapa socialista. Afirmar que "de cada uno según su capacidad, a cada uno según su necesidad" describe la superioridad ética del principio solidario sobre el principio meritocrático. Este último tiene un propósito estrictamente utilitario: ampliar el bienestar colectivo (la familia, en este caso). Sugiere que el mérito enciende el esfuerzo; es la tarea productiva. Pero se frena subordinándolo al criterio solidario. Es el momento de la distribución según las necesidades. Y esto es lo que les sucede a las familias: sus miembros, incluidas las personas en infancia y en condición de vejez, obtienen su porción de acuerdo con sus necesidades, no en función de sus capacidades.

La solidaridad y los criterios meritocráticos son analíticamente diferentes. Pero el hecho de que las personas respondan de la misma manera ("no") a las preguntas asociadas, revela la coexistencia en cada persona de dos sentidos de justicia contradictorios. Forman un equilibrio armonioso que ha permitido a la modernidad avanzar dentro de límites claros. Como ha sugerido el neoinstitucionalismo sin dosis limitadas, más bien estratégicas de premodernidad, la modernidad y la economía capitalista no podría funcionar (North, 1993).

El capitalismo tradicional respetó ese equilibrio durante mucho tiempo. La inmunidad familiar ante los vaivenes del mercado es una buena prueba. El estado de bienestar muestra la misma lógica: la provisión de pensiones refleja la esencia de la solidaridad sistemática: ayuda destinada a quienes no pueden afrontar por sí mismos los desafíos cruciales de la vida. La fórmula del sistema de pensiones era una forma diferente de reproducir lo que esta- 
ba sucediendo, y esperábamos que sucediera, en el núcleo familiar. Las personas mayores apoyaron a los niños y niñas cuando eran pequeños, al igual que los niños/las niñas se ocuparán de los ancianos cuando no tengan la fuerza para trabajar. Y lo ocurrido en la familia también evocaba los límites en el criterio meritocrático: si no es posible que una persona haga su propio esfuerzo, los demás son responsables de su bienestar.

La llegada del neoliberalismo lo cambió todo. No es casualidad que viniera con el fenómeno cultural del posmodernismo, que se caracteriza por el hiperindividualismo, el imperio del hedonismo y, principalmente, por lo que Lipovetsky (2001) denominó “el ocaso del deber”. La familia perdió no solo su naturaleza nuclear; También se asemejó a esa etapa de la modernidad: líquida, en expresión de Bauman (2005 y 2006). Ambos, entre amigos y familiares, los lazos se volvieron efímeros; circunscrito al tiempo y al espacio (mientras el trabajo, el amor, la armonía... duren). El único vínculo permanente es el que existe entre una madre y sus hijos e hijas. Indisoluble, pero asimétrico: emerge el príncipe-niño y sus caprichos gobiernan la dinámica del hogar. Todo lo demás es cambiante y el cambio es legítimo: amigos, parejas.... todos eran de usar y tirar. El ego se convirtió en el referente imperativo, y los demás, un accidente o una circunstancia temporal y desechable. Todo lo que da placer y consuelo es bienvenido como parte de su vida. Todo lo que lo incomode, requiera un esfuerzo y obstruya nuestros planes y deseos debe tener una fecha de vencimiento. Por tanto, se pierde el sentido del deber. El deber hacia los demás, hacia su país, hacia el cuerpo. Mientras que el humanismo renacentista sugirió que "el hombre era la medida de todas las cosas", el individualismo posmoderno reformula: "Yo soy la medida de todas las cosas".

Este cambio cultural refleja la profunda mutación que han sufrido las subjetividades. Sería una exageración claramente desproporcionada afirmar que las nuevas subjetividades fueron las que trajeron cambios al sistema de pensiones. Sin embargo, sí crearon un ambiente propicio para que el neoliberalismo pudiera alterar el mecanismo de reciprocidad que, a partir de la familia, reproducía el sistema de distribución. Representaba, sobre todo, la legitimación del individualismo sin restricciones. Era exactamente lo que necesitaba el neoliberalismo, en la medida en que la cultura daba un significado relativo y subordinado al sentido de la solidaridad. Entonces, la base del estado de bienestar fue cancelada porque este nuevo significado hizo desaparecer la característica natural del deber moral. El cambio cultural facilitó lo que se venía de- 
sarrollando en los campos de la economía, la política y la convivencia social: la llegada de una nueva etapa del capitalismo: el neoliberalismo.

La esencia de todo tipo de liberalismo es hacer del bienestar una responsabilidad exclusivamente individual. El neoliberalismo recoge, en sus términos, esta consigna. "Neo" porque vuelve cuando se pensaba que el estado del bienestar había llegado para quedarse y que la solidaridad finalmente había demostrado ser el componente esencial para traer la cohesión social que el mercado no puede alcanzar. Pero es "liberal" porque pretende invadir, con su lógica individualista, todas las dimensiones de la vida social. La ambición de gobernar los sistemas de educación y salud a través de váuchers que estimulaban la competencia entre las instituciones encargadas de proveerlos, ya fueran privados o públicos, constituía uno de los ejemplos más elocuentes de la intención liberal de convertir en mercancía todas, o casi todas, las esferas sociales. Se intentó imponer una lógica similar para carreteras, puentes, caminos de entrada y salida de ciudades, cárceles, agua, etc. Todo estaba sujeto al comercio, por la vía privada significaba, supuestamente, la mejor gestión de los servicios públicos. Esa fue, invariablemente, la racionalización de los abogados del neoliberalismo, la eficiencia en el sentido de Pareto: el libre mercado como mecanismo insuperable a la hora de impulsar el bienestar social (Stiglitz, 2000). Pero, como señala Polanyi (1944/2001), la entronización del libre mercado fue un hecho que contradecía la historia de la humanidad, en términos de que desligó la economía de la vida misma, en la que había estado "incrustada". De repente cobró vida, independizándose de otras esferas vitales, hasta el punto de dominar cada uno de sus rincones.

La llegada del sistema de pensiones de capitalización individual anuncia la transgresión de un ámbito que hasta entonces era sagrado: el bienestar de las personas mayores. Cada uno se hizo responsable de su propio bienestar, del futuro, pero también del presente.

La gente no podía esperar que las generaciones venideras desplegaran los sacrificios y la reciprocidad que la tradición había impuesto. El diseño institucional del nuevo sistema no lo permitió. Los incentivos asociados se estructuraron para romper cualquier forma de solidaridad intergeneracional, no solo entre la sociedad y sus mayores (que habían contribuido a construir), sino también entre familiares de diferentes edades. Un hombre en el apogeo de su edad laboral debería planificar, al mismo tiempo, su futura jubilación y el bienestar de sus dependientes económicos. Por un principio básico de 
escasez, no podía "servir a dos amos". Se rediseñan las dinámicas familiares para activar un juego de suma cero: los recursos dedicados al futuro personal deberían traducirse en menos recursos destinados al resto de la familia; y a la inversa. Pero en las comunidades rige un principio de reciprocidad: los jóvenes pagarán por el bienestar de las personas mayores como lo hacían cuando eran niños y niñas. Este principio fue respetado en la etapa de la modernidad, pero ahora está en serio peligro bajo la posmodernidad, debido a la relación asimétrica entre padres e hijos, ligada al cambio de subjetividades intergeneracionales que la cultura trajo consigo.

En las sociedades ricas, estas subjetividades asimétricas no acarrean mayores consecuencias, pero el dilema se agravó en los países pobres y subdesarrollados, en los que los salarios y la renta per cápita son muy bajos. Además, cuando se activa la política neoliberal de apertura externa en un contexto de baja competitividad, la depresión salarial se convierte en una herramienta obligatoria para enfrentar los desafíos de la competencia externa. Se instituyó así una clara estrategia de depresión salarial, que agravó el dilema personal.

Las familias que viven en estos países a menudo se rigen por estándares tradicionales. Entre ellos, el que prescribe el sacrificio de los padres por el bien de sus hijos, fue predominante entre quienes habían vivido su infancia bajo esa tradición. Y, antes de mostrar cualquier tipo de egoísmo en detrimento de sus hijos, aceptan sacrificios, aunque ellos no serán compensados. El resultado puede ser una pensión de pobreza legitimada por la asimetría de subjetividades intergeneracionales.

Germani (1962) denominó “efecto fusión” a la propensión de los países en desarrollo a adoptar modas culturales nacidas y cultivadas en las naciones ricas. Esto sucede en México: la mayoría de la juventud contemporánea en edad laboral no cree en los valores tradicionales. Más inclinados a la cultura de la posmodernidad del hiperindividualismo, dan mayor valor al "culto al ego" sobre cualquier otro tipo de obligación, especialmente las de carácter ético. Para ellos, la moral ha perdido su carácter absoluto y se relativiza con la comodidad personal y el bienestar propio. La consecuencia es que las reglas informales que hicieron posible el sacrificio paterno, probablemente no serán repetidas por sus hijos con el mismo sentido de reciprocidad. La cultura posmoderna rompió con ese sentido y tiende a instrumentalizar las relaciones personales, incluidos los lazos familiares. Eso explica la abundancia de hogares de ancianos y su abandono; padres que no son visitados por sus hijos; impaciencia ante las dificultades para aprender a gestionar las 
nuevas tecnologías, etc. Esto significa que el neoliberalismo ha encontrado en la posmodernidad un compañero de viaje especialmente útil. Entonces, la pobreza de las pensiones puede legitimarse.

En América Latina, existe una clara tendencia a volver a los esquemas de pensiones de carácter solidario. Esa tendencia significa, no la cancelación de la capitalización individual, sino su redefinición como esquema optativo y complementario del sistema solidario, que vuelve a convertirse en el pilar de las pensiones.

Esta restauración va de la mano de la crisis teórica y ética que vive el neoliberalismo. Este fue el cambio que profanó lo que antes era sagrado: la extrapolación de la lógica familiar hacia ciertas dimensiones sociales comunitarias. El capitalismo tradicional había respetado esta esfera manteniéndola al margen de cualquier intento de mercantilización. Pero el neoliberalismo rompió las barreras protectoras e incluyó prácticamente todas las esferas de la vida dentro de la esfera del mercado. Las pensiones no escaparon a la ola invasora y se dejaron seducir por el espíritu del liberalismo: la responsabilidad individual del bienestar y la obsolescencia de lo que las comunidades tradicionales habían dado a la convivencia social moderna: la solidaridad de todos en aquellos aspectos que son esenciales para una vida digna.

La ruptura de tal solidaridad se ha manifestado en la legitimidad de la que aún disfruta el modelo meritocrático en algunas partes. En el caso de México - que ha sido elegido como laboratorio para presentar estas ideas centrales - se muestra como indiferencia hacia la tendencia de la movilidad social descendente de los jubilados — en el escenario más optimista - y hacia la pobreza de los jubilados, bajo el más realista.

\section{Bibliografía}

Alonso, J., Hoyo, C., \& Tuesta, D. (2014). Un modelo para el sistema de pensiones en México: diagnóstico y recomendaciones. Documento de trabajo 14/17. BBVA. http://bitly.ws/e5ew

Arenas, A. (2019). Los sistemas de pensiones en la encrucijada. Desafío para la sostenibilidad en América Latina. CEPAL, Santiago de Chile. bit. ly/3uh5IAybit.1

Bauman, Z. (2005). Amor líquido. Acerca de la fragilidad de los vínculos humanos. Fondo de Cultura Económica. 
Bauman, Z. (2006). La modernidad líquida. Fondo de Cultura Económica.

Banco Mundial (BM9, Banco Interamericano de Desarrollo (BID), OCDE (Organización para la Cooperación y Desarrollo Económica) (2015). Panorama de las pensiones: América Latina y el Caribe. https://bit.ly/3aXBr1Y

Clavellina, J. (2020). Sobre la reforma al sistema de pensiones en México. Pluralidad y consenso, 10(45). Instituto Belisario Domínguez, Senado de la República. http://bitly.ws/e57B

Comisión Nacional del Sistema de Ahorro para el Retiro (CONSAR) (2018). ¿De cuánto serán las pensiones en México?: un análisis de sensibilidad de la cuenta Afore ante cambios paramétricos. CONSAR, Documento de trabajo \# 9 Ciudad de México, México

Comisión Nacional del Sistema de Ahorro para el Retiro (CONSAR) (2020). El SAR en números https://bit.ly/3vFyISG

Durán, F., \& Peña, H. (2011). Determinantes de las tasas de reemplazo de pensiones de capitalización individual: escenarios latinoamericanos comparados. CEPAL.

Durkheim, E. (1964). The Division of Labor in Society. Free Press.

Filgueira, F., Molina, C. G, Papadópulus, J., \& Tobar, F. (2006). Universalismo básico: una alternativa posible y necesaria para mejorar las condiciones de vida. En Carlos Gerardo Molina (Ed.), Universalismo básico. Banco Interamericano de Desarrollo y Planeta. Washington. https://bit. ly/3nRQ64c

Herrera, G., \& Velázquez L. (2018). Más viejos, menos pensiones. Resultados de las reformas estructurales al sistema de pensiones en México (1995-2014). En C. Bayón, Sara Ochoa y José Guadalupe Rivera González (Coords.), Desigualdades, pobreza, economía informal, precariedad laboral y desarrollo económico. Vol. III. COMECSO. http://bitly.ws/e5gg

Germani, G. (1962). Política y sociedad en una época de transición, de la sociedad tradicional a la sociedad de masas. Editorial Paidos.

Instituto Mexicano del Seguro Social (IMSS). (2020). Datos abiertos. https://bit. ly/2RmHJkO

Instituto Nacional de Estadística, Geografía e Informática (INEGI) (2020). Encuesta Nacional de Empleo y Ocupación, agosto. https://bit.ly/3eSrIuV

Lipovetsky, G. (2001). El crepúsculo del deber. La ética indolora de los nuevos tiempos democráticos. Anagrama.

Martínez-Preece, M., Sosa, M., \& Zubieta-Badillo, C. (2019). Dinámica y desempeño de los fondos de pensión en México (1997-2018): un análisis de 
volatilidad condicional con cambios estructurales. Revista de Economía, 36(93). Facultad de Economía, Universidad Autónoma de Yucatán. http://bitly.ws/e5dV

North, D. (1993). Instituciones, cambio institucional y desempeño económico. Fondo de Cultura Económica.

Ortiz, I., Durán-Valverde, F., Urban, S., \& Wodsak, V. (Eds.) (2018). Reversing pension privatizations: Rebuilding public pension systems in Eastern Europe and Latin America. ILO. http://bitly.ws/e5eP

Organización para la Cooperación y Desarrollo Económica (OCDE) (2016). Estudio de la OCDE sobre los sistemas de pensiones: México, versión en español. CONSAR. Ciudad de México, México. https://bit.ly/3xMhJQq

Polanyi, K. (1944/2001). The great transformation: the political and social origins of our time, 2nd ed., Beacon Press.

Ramírez, C. (2017). Reforma de pensiones en México: avances, logros y retos. El Cotidiano, núm. 204, julio-agosto, pp. 29-39, Universidad Autónoma Metropolitana Unidad Azcapotzalco. http://bitly.ws/e5ft

Rodríguez, K. (2016). La pobreza de los adultos mayores y la operación de la provisión social en México: principales problemáticas y los cambios necesarios. O Social em Questão, 19, 105-122. http://bitly.ws/e5ha

Sánchez, P., \& Rodríguez, K. (2020). Pensiones no contributivas en México y pobreza de los adultos mayores (PAM) 65 y más en 2018. Revista Euro Latinoamericana de Análisis Social y Político RELASP, 1(2). http://bitly.ws/e5dk

Secretaría de Hacienda y Crédito Público (2020). Presentación del secretario de la propuesta para las pensiones. https://bit.ly/3ujkkiN

Stiglitz, J. (2000). Economics of the public sector. Norton \& Company.

Villarreal, H., \& Macías, A. (2020). El sistema de pensiones en México: institucionalidad, gasto público y sostenibilidad financiera. Serie Macroeconomía del Desarrollo, N² 210 (LC/TS.2020/70), Comisión Económica para América Latina y el Caribe (CEPAL). Santiago. http://bitly.ws/e572 